\title{
Enzymatic Glyceride Synthesis in a Foam Reactor
}

\author{
Yu-Chih Yeh and Erdogan Gulari* \\ Department of Chemical Engineering, University of Michigan, Ann Arbor, Michigan 48109
}

\begin{abstract}
We report the results of our study on Rhizomucor miehei lipase-catalyzed lauric acid-glycerol esterification in a foam reactor. A satisfactory yield of glyceride synthesis can be achieved with an unusually high initial water content $(50 \% \mathrm{w} / \mathrm{w})$. We found that product formation could be regulated by controlling foaming. Foaming was a function of the air flow rate, reaction temperature, $\mathrm{pH}$ value, ionic strength, and substrate molar ratio. Monolaurin and dilaurin, which constituted nearly $80 \%$ of the total yield, were the two dominant products in this reaction; trilaurin was also formed at the initial stages of the reaction. A study of $\mathrm{pH}$ and ionic strength effects on an independent basis revealed that they affect the interfacial mechanism in different manners. On varying the ratio of lauric acid and glycerol, only a slight change in the degree of conversion was detected and the consumption rate of fatty acid was approximately the same.

JAOCS 75, 643-650 (1998).
\end{abstract}

KEY WORDS: Esterification, foams, glyceride synthesis, HPLC, lipase, Rhizomucor miehei, substrate inhibition.

Lipases have been successfully employed in catalyzing many organic reactions (1-5). Among those reactions, lipase-catalyzed esterifications are of most commercial interest (6-8). For instance, esterification of sugar with fatty acids produces sugar esters, which are potentially important as food and cosmetic emulsifiers (9). A substitute for a natural wax ester, jojoba oil, can be derived from the transformation of high-erucic acid rapeseed oil and erucyl alcohol (10). The main products of the reaction discussed in this paper, mono- and diglycerides, are extensively used in food and cosmetic additives to increase the flavor and enhance emulsifying as well as texturizing functions. The use of lipases has broadened over the last decades to include the synthesis of biosurfactants, cloning of a detergent lipase (11), and biorefining of oils (12).

Enzyme-catalyzed reactions, as an alternative to traditional chemical synthesis, have become increasingly important in the food, cosmetics, and pharmaceutical industries because, in general, no toxic materials are involved, and reaction conditions are mild and have high stereoselectivity (13-15) as well as enantioselectivity, which is advantageous for the production of fine chemicals. Though lipase is active at oil/water interfaces, immiscibility of oil (substrates and products) and water (essential for enzymatic function) complicates the esterification process. As a result, a lot of effort

*To whom correspondence should be addressed. E-mail: Gulari@engin.umich.edu. has been spent to enhance the oil/water contact area (and therefore the catalytic efficiency of the lipase) by designing an optimal reaction environment to carry out this reaction.

Generally, reaction media for esterification by lipases can be classified into two categories, organic and solvent-free media (in aqueous solutions, the equilibrium is shifted far to the hydrolysis side; thus, aqueous solutions are not suitable for esterification). When an organic medium is employed, organic solvents have to be used to dissolve the reactants. By magnetic stirring $(14,15)$, using immobilized lipase (16) or substrate support $(17,18)$, or adding surfactants to form micelles (19-21), the interfacial area between lipids and the aqueous phase can be increased considerably. However, drawbacks include mass transfer limitations, need for toxic solvents and surfactants in the separation step, and difficulty of operating reactions in a continuous mode. In contrast, reactions carried out in a solvent-free medium do not cause contamination, but have other disadvantages. When incubating substrates and lipase together in a nonaqueous medium (22) or performing reactions in a membrane reactor $(23,24)$, high viscosity may lead to enzyme denaturation, and scale-up problems may be serious. Recently, high yields of monoglycerides and diglycerides have been achieved by temperature programming of enzymatic glycerolysis of fats (25-30). In spite of high conversion and high purity, lowering reaction temperature may result in a sharp decline in reaction rates, and the solidified reaction mixture may complicate the separation of products and lipase. This method is not suitable for glycerides of monounsaturated or polyunsaturated fatty acids, owing to their low melting points.

As a consequence, there still exists a need to develop a reaction environment for lipase that optimizes the catalytic efficiency at oil/water interfaces. It is well known that the incubation conditions as well as the reaction microenvironment have a governing effect on enzymatic mechanisms, in that the catalyzing pattern may differ as the interfacial property changes. In this paper, we report the effects of various factors on the enzymatic glyceride synthesis in a foam reactor. Because lipase and most of the reactants possess sufficient surface activity, there is no requirement of adding surfactants or solvents, thereby vastly reducing the cost of down-stream processing. In this work, we report our results obtained with a new reaction medium, foams, to host the fatty acid-glycerol (GLY) esterification catalyzed by lipase from Rhizomucor miehei.

Several methods are available to make foams (31). All of them involve a large amount of gas being introduced into a 
liquid, during which more and more gas bubbles are produced and several changes start to occur (31):

(i) Disproportionation or Ostwald ripening: as time increases, bigger bubbles grow in size by diffusion of the gas through the liquid continuous region at the expense of smaller bubbles;

(ii) Creaming: because air bubbles are buoyant and fairly large, they cream rapidly by forming a separate foam layer on top of the bulk liquid;

(iii) Polyhedral geometry: as more bubbles are formed, the bubble cells come in contact with and deform one another, leading to foam cells of polyhedral shape, which minimizes the total surface area. Each of these cells has 12 neighbors. At this stage, the structure of the foams consists mainly of a large number of thin liquid films (lamellae), which make an angle of $120^{\circ}$ with one another. The line where the thin films meet is called the Plateau border, which encloses most of the liquid in the foams $(31,32)$;

(iv) Drainage: owing to gravitational effects, the central liquid between the foam cells starts to drain from the Plateau borders to bulk liquid;

(v) Rupture or formation of stable black film: at the final stage of foam drainage, the film thickness reaches an approximate equilibrium value of several hundred Ångstroms. At more than a minimum concentration of the solute, a black film is formed after drainage, which indicates that its thickness is less than the wavelength of light. Alternatively, the films often become more brittle with draining and can resist no more stress, resulting in film rupture and bubble coalescence.

By bubbling air through a reaction mixture that contains lipase, GLY, water, and a fatty acid, foaming can be induced. It is our goal to relate the hydrodynamics of the foam system to the catalytic performance of the lipase. The formation of numerous air/liquid thin films increases the contact area between the oil and aqueous phases significantly. Furthermore, with most of the liquid phase (mainly GLY and buffer) and excess substrates being in the Plateau borders, the reaction equilibrium on foam films can be driven toward glyceride synthesis.

\section{EXPERIMENTAL PROCEDURES}

Materials. All reactants, such as lauric acid (LA) and GLY, were obtained from Sigma Chemical Co (St. Louis, MO). Monolaurin (ML), dilaurin (DL), and trilaurin (TL), as standards for high-performance liquid chromatography (HPLC) calibration, were purchased from Sigma at the highest purities available. The solvents (Fisher Scientific, Pittsburgh, PA) for HPLC analysis (chloroform, acetone, and acetonitrile) were used without further purification. Lipozyme, a fungal lipase in liquid form (activity $10 \mathrm{LU} / \mathrm{mg}$ of solution), from $R$. miehei, was a generous gift from Novo Industry (Copenhagen, Denmark). Various buffer solutions with different ionic concentrations and the phosphate salts used in preparing phosphate buffers of different $\mathrm{pH}$ values were all obtained from Sigma Chemical Co.
Product analysis. To achieve a good separation of fatty acid and glycerides, HPLC was used (33) with a Waters (Milford, MA) ALC 200 Series, WISP sample injector, and Data Module. The stationary phase was the Supelcosil ${ }^{\mathrm{TM}}$ LC-18 column from Supelco Company (Bellefonte, PA). The mobile phase was a mixture of acetone and acetonitrile at a 1:1 volume ratio at a constant flow rate of $0.8 \mathrm{~mL} / \mathrm{min}$. For each analytical run, a $15-\mu \mathrm{L}$ sample was injected. The duration for one analysis was $45 \mathrm{~min}$, with an additional 10 min needed to resume the stability of the chromatographic system. In this work, the reverse-phase method was employed. An evaporative light-scattering detector (ELSD IIA; Varex Corporation, Rockville, MD) was installed on the HPLC to monitor the eluting components from the chromatographic column $(34,35)$. ELSD operating conditions were as follows: drift tube temperature and exhaust temperatures of 100 and $80^{\circ} \mathrm{C}$, respectively, and a carrier gas flow of $1 \mathrm{~L} / \mathrm{min}$ dry nitrogen. The selectivities are expressed as mol\% of the starting fatty acid contained in each specific product (ML, DL, unreacted fatty acid, etc.) at the time of sampling. Direct measurement of the LA concentration with the light-scattering detector was not reliable, and all lauric concentrations reported are calibrated by mass balance from the measured concentrations of the mono-, di-, and triglycerides.

Foam reactor. Figure 1 is a schematic diagram of the foam reactor within which lipase-catalyzed esterifications were conducted. The inner diameter of this reactor was $3 \mathrm{~cm}$, and a porous plate was located $3 \mathrm{~cm}$ above the bottom to evenly distribute the incoming dry air into the reaction liquid, thereby producing the foams. A water jacket with a thickness of $2 \mathrm{~cm}$ surrounded the reactor to control the temperature.

Enzymatic reactions. An initial 50\% (w/w) of nonionic or buffered solution and reactants (fatty acid and GLY), at different molar ratios, was first incubated in the reactor for $0.5 \mathrm{~h}$ to allow the fatty acid to melt and the whole system to be thermally equilibrated. To avoid overflow of foam, the total liquid volume was adjusted to approximately $6 \mathrm{~mL}$. In all experiments, the GLY was present in excess, and its amount was held constant. The most common molar ratio used was $\mathrm{LA} / \mathrm{GLY}=0.1$, corresponding to a molar concentration of $0.56 \mathrm{M}$ for LA at the beginning of the reaction. The starting molar concentration of GLY was kept constant at 5.6 M. The starting water concentration was also constant at $27 \mathrm{M}$; however, this dramatically decreased toward the end of the reaction. The final water contents of the reaction mixtures were determined by a Mettler-Toledo (Worthington, OH) DL18 Karl Fischer auto titrator.

After the start of air flow, esterification reactions were initiated by addition of $0.04 \mathrm{~mL}$ of unpurified lipase-containing protein solution for each $\mathrm{mL}$ of the starting reactant mixture. The amount of lipase in the protein solution is given as 10 LU/mg of liquid by the manufacturer. We did not check this independently. Owing to the surface activity of both fatty acid and enzyme molecules, bubbles of uniform size were immediately formed. At regular intervals, $200 \mu \mathrm{L}$ of foam materials was sampled in a capillary tube and diluted with chloroform to 


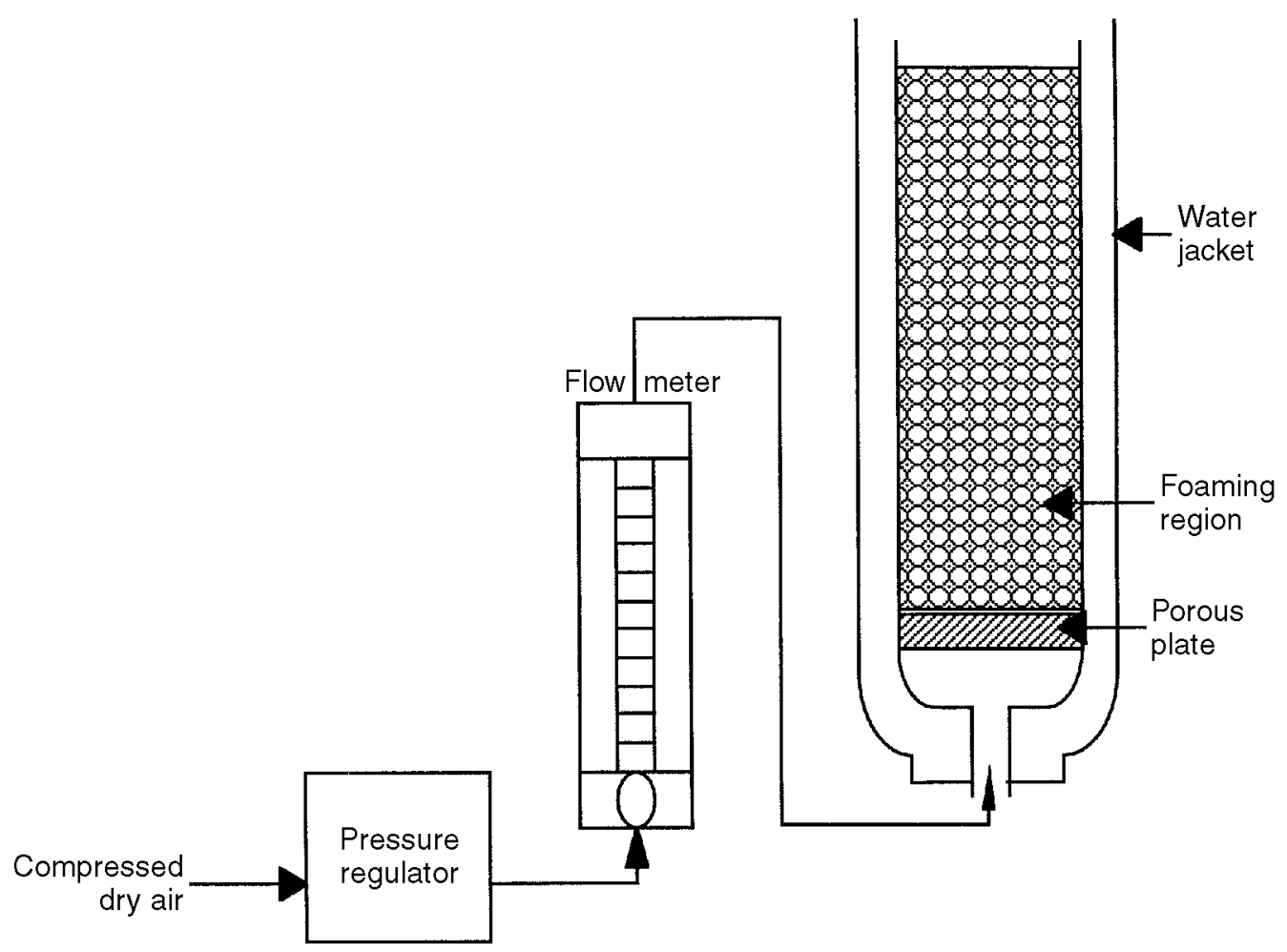

FIG. 1. Schematic diagram of the foam reactor used for this study.

a concentration range appropriate for HPLC analysis. To determine the reproducibility of our results, we performed several duplicate runs. Overall, the results were reproducible to better than $\pm 10 \%$. GLY, initially added in excess, was not monitored during the reaction. Hence, chloroform, a good solvent for all other reactants except GLY, was the ideal candidate as a solvent for the following HPLC analysis. All reactions were stopped after $24 \mathrm{~h}$ owing to reduction in foam density.

The concentrations of the products were calculated and are reported in terms of fatty acid equivalents (i.e., the total amount of the fatty acid converted to a monoglyceride is equal to the molar concentration of the monoglyceride; for a diglyceride, the fatty acid equivalent is twice the molar concentration of the diglyceride, and for a triglyceride it is three times).

\section{RESULTS AND DISCUSSION}

Effect of flow rate. Figure 2 shows product distribution and overall conversion for a representative set of conditions. At medium air flow rates such as $20 \mathrm{~mL} / \mathrm{min}$ \{corresponding to a superficial velocity of $2.8 \mathrm{~cm} / \mathrm{min}\left[=\left(20 \mathrm{~cm}^{3} / \mathrm{min}\right) /(\right.$ column cross-sectional area of $\left.\left.\left.7.06 \mathrm{~cm}^{2}\right)\right]\right\}$ and $25 \mathrm{~mL} / \mathrm{min}$, the amounts of ML and DL were almost always equal within experimental error. The final composition of the products (as mol\% of the fatty acid converted to each product) after $24 \mathrm{~h}$ was $49 \% \mathrm{ML}, 40 \% \mathrm{DL}$, and $11 \%$ TL. The change in the final conversion as a function of air flow rates is shown in Figure 3 . We also noted that the rate of water removal was a strong function of the air flow rate. For $20 \mathrm{~mL} / \mathrm{min}$ flow rate, the water content of the reaction mixture decreased linearly to $22 \%$ by weight at the end of a $24-\mathrm{h}$ run; for $25 \mathrm{~mL} / \mathrm{min}$ flow rate, the final concentration was $4 \%$ by weight. Though lower yields were obtained at higher or lower air flow rates, at least

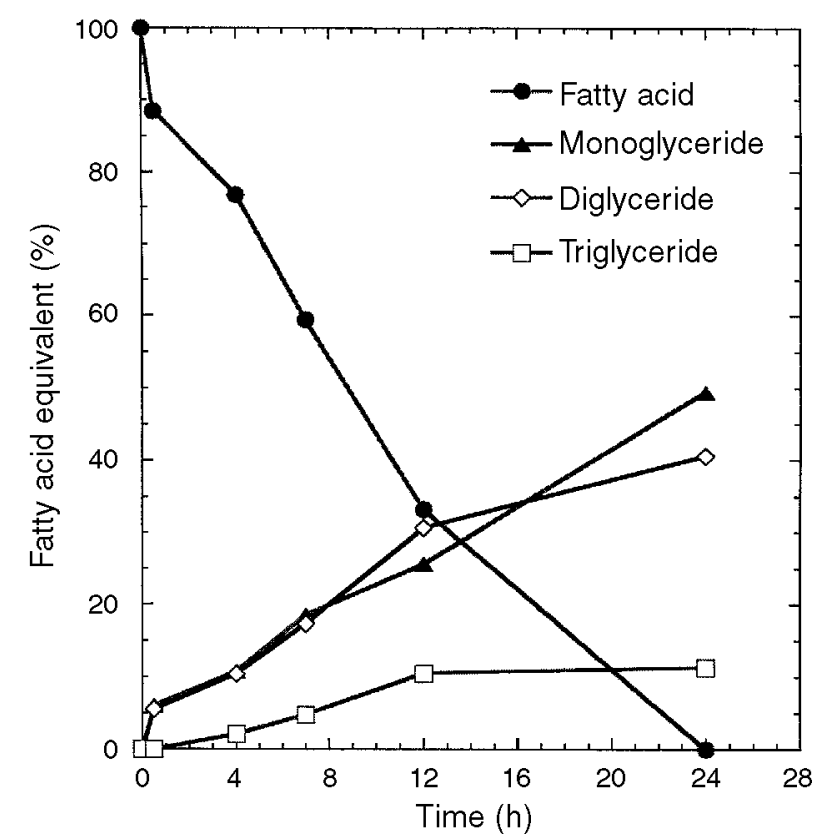

FIG. 2. Results for a representative set of conditions for lauric acid/glycerol (LA/GLY) esterification with a substrate molar ratio of $L A / G L Y=0.1$, $\mathrm{T}=50^{\circ} \mathrm{C}$, flow rate of $25 \mathrm{~mL} / \mathrm{min}$, and $\mathrm{pH} 7$ (0.1 M phosphate buffer). 


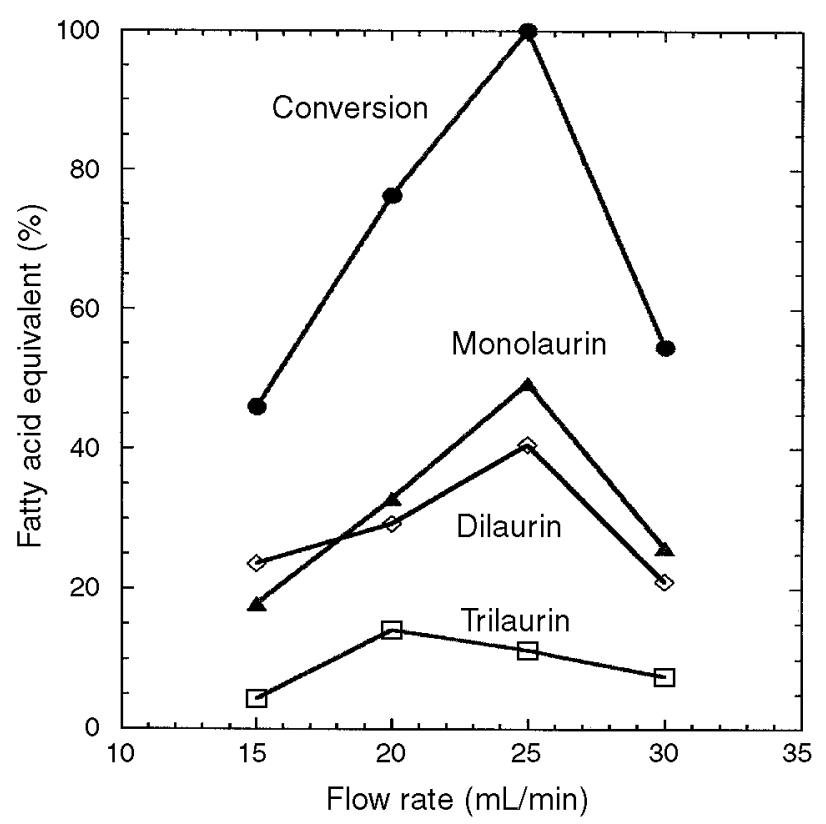

FIG. 3. Effect of gas flow rate on the final composition after $24 \mathrm{~h}$ of reaction. The reaction conditions are the same as those of Figure 2.

$50 \mathrm{~mol} \%$ of LA was converted within $24 \mathrm{~h}$, with the main products being $\mathrm{ML}$ and DL. At a flow rate of $15 \mathrm{~mL} / \mathrm{min}$, a lower reaction rate and larger initial fluctuations in product formation (data not shown) were observed. The reason may be that the reactants were not fully mixed initially, and that water was not efficiently removed during the reaction. Nonetheless, good foaming conditions were maintained throughout the reaction course, and DL was present in a larger amount (fatty acid molar equivalents) compared to ML. In contrast, reactions carried out at higher flow rates gave slightly higher concentrations of ML than DL. At high flow rates, foaming was relatively unstable, and the foam region appeared to be shorter than those obtained at lower air flow rates. Within the air flow rate range tested, the optimum flow rate for reactions with a substrate ratio ([LA]/[GLY]) of 0.1 was between 20 and $25 \mathrm{~mL} / \mathrm{min}$ during which the conversion of LA ranged from 76 to almost $100 \%$ after $24 \mathrm{~h}$.

The optimal operating range may, however, vary with reaction conditions. For instance, a maximum conversion of nearly $75 \%$ was observed at a flow rate of $30 \mathrm{~mL} / \mathrm{min}$ when the molar ratio of LA to GLY was increased to 0.2.

We speculate that foaming is a controlling factor for glyceride synthesis. The primary reason is that, with good foaming, the interfacial area is increased and lipase and the reactants have a larger probability of interaction to form the acylenzyme intermediate.

Temperature effect. The effect of temperature on the esterification reaction at different substrate molar ratios is illustrated in Figure 4. At a low substrate molar ratio ([LA]/[GLY] $=0.1$, conversions did not vary considerably within the temperature range of 50 to $70^{\circ} \mathrm{C}$. ML and DL were again the two dominant products. Figure 4A shows that there was a loss of
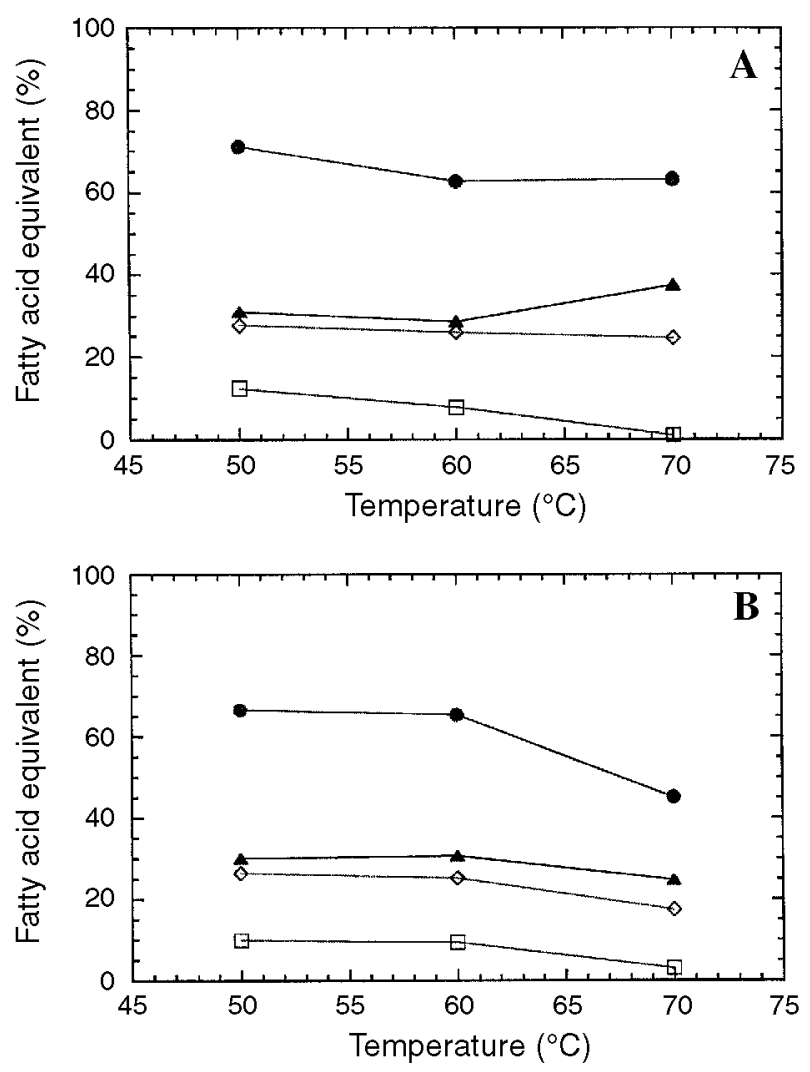

FIG. 4. Effect of temperature on the final product distribution of reactions for different substrate molar ratios: $(\mathrm{A}) \mathrm{LA} / \mathrm{GLY}=0.1$; (B) $\mathrm{LA} / \mathrm{GLY}$ $=0.2$; phosphate buffer solution of $\mathrm{pH} 8$. Other conditions, symbols, and abbreviations are the same as Figure 2.

TL at higher temperatures, which was compensated by higher ML production. However, Figure 4B shows that, with a higher substrate molar ratio $(\mathrm{LA} / \mathrm{GLY}=0.2)$, the conversion decreases by $\sim 50 \%$. This decrease is seen in all three products. Within the temperature and substrate ratio ranges tested, the lipase remained active and gave maximum conversion of $\sim 70 \%$ after $24 \mathrm{~h}$. From the detailed reaction courses shown in Figures 5A and 5B, we observe that ML and DL are formed almost at the same rate (in terms of fatty acid equivalent mol\%) during the first $6 \mathrm{~h}$; nonetheless, DL was the favored product to be formed during the first 12 to $20 \mathrm{~h}$ at $50^{\circ} \mathrm{C}$ (Fig. $5 \mathrm{~A})$, whereas ML prevailed when reactions were carried out at $70^{\circ} \mathrm{C}$ (Fig. 5B). Figure 5B shows that, at $70^{\circ} \mathrm{C}$, little fatty acid is consumed after $12 \mathrm{~h}$, and there is almost no TL produced. This phenomenon was also true for reactions with higher substrate molar ratios (data not shown).

The cessation of conversion (mainly of TL) at higher temperatures could be due to two factors. First, as water was lost to dry air, the reaction mixture became so viscous that the reaction mixture did not foam anymore. As a result of the decreased interfacial area, the probability of interfacial interaction between ML and the enzyme was less, and further conversion of ML stopped. This was in accordance with the observation reported above for the flow rate effect. Second, some limited lipase denaturation may happen, especially 

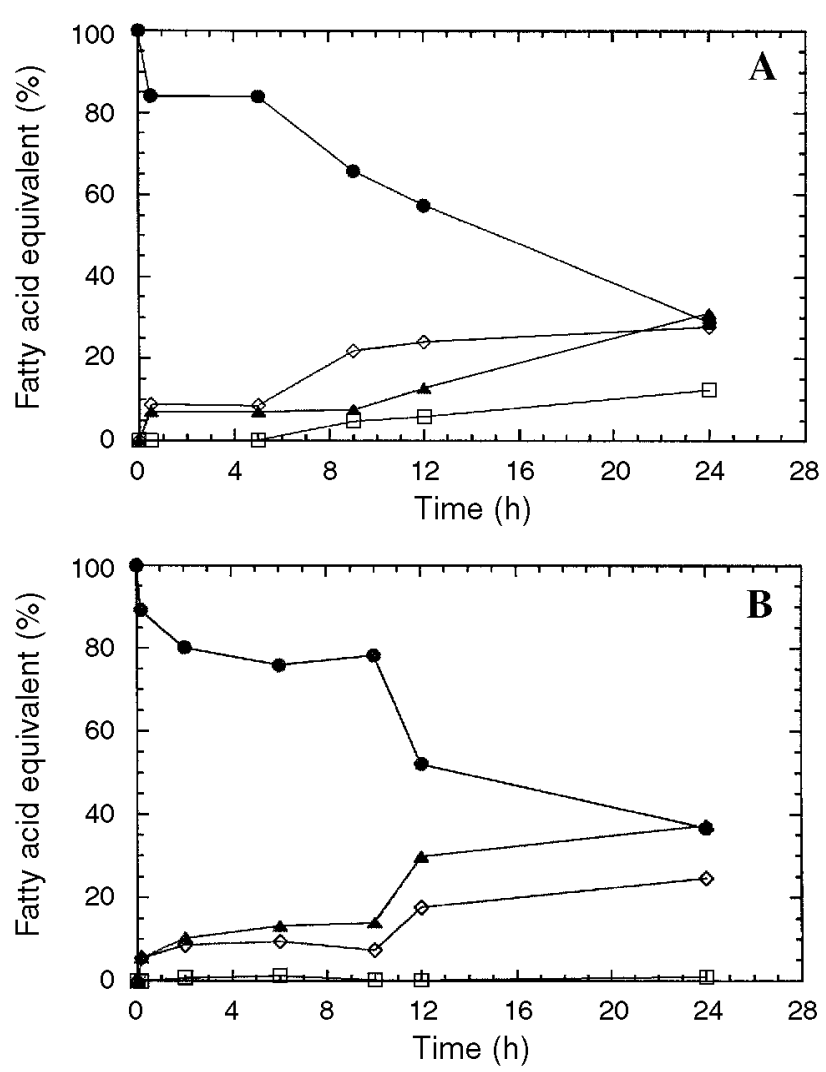

FIG. 5. Effect of temperature on the esterification of GLY with LA; (A) T $=50^{\circ} \mathrm{C}$, and $(\mathrm{B}) \mathrm{T}=70^{\circ} \mathrm{C}$. Reaction conditions: Gas flow rate: 20 $\mathrm{mL} / \mathrm{min}$, and $0.1 \mathrm{M}$ phosphate buffer of $\mathrm{pH}$ 8. Symbols and abbreviations are the same as in Figure 2.

when enzymes are exposed to high temperatures and viscous media for a prolonged duration. Finally, the higher temperatures increase the rate at which equilibrium is reached.

Effect of $\mathrm{pH}$. Figure 6 shows that when $\mathrm{pH}$ was increased from 5 to 8 , conversion also increased from 30 to $70 \%$. The conversion was low when reactions were carried out at a $\mathrm{pH}$ of 5 or 6 where foaming was poor. On the contrary, the highest conversion was obtained at $\mathrm{pH} 8$, at which the best foaming was observed. With a Tris buffer, the foam materials overflowed in a crystallized form at $\mathrm{pH}$ 9. Owing to the difficulty in sampling, we did not carry out experiments at $\mathrm{pH}$ values higher than 8. However, the conversion data shown in Figure 6 indicate that it may be possible to reach even higher conversions at starting $\mathrm{pH}$ values above $\mathrm{pH} 8$ if foaming can be controlled.

At alkaline $\mathrm{pH}$, the free fatty acid is converted to a salt form (a soap), which stabilizes the foam. Thus, $\mathrm{pH}$ affects not only the activity and stability of the enzyme but also changes the amount of interfacial area available. Thus, it is not surprising to find increased conversions at higher $\mathrm{pH}$ values. In our subsequent studies, we found that the interaction between the lipase and the ionic lipid layer is $\mathrm{pH}$ dependent.

Both $\mathrm{pH} 7$ and $\mathrm{pH} 8$ are good $\mathrm{pH}$ environments for lipase. Though reactions at $\mathrm{pH} 8$ had higher conversions, the main difference was in the yield of TL. For enzymatic reactions

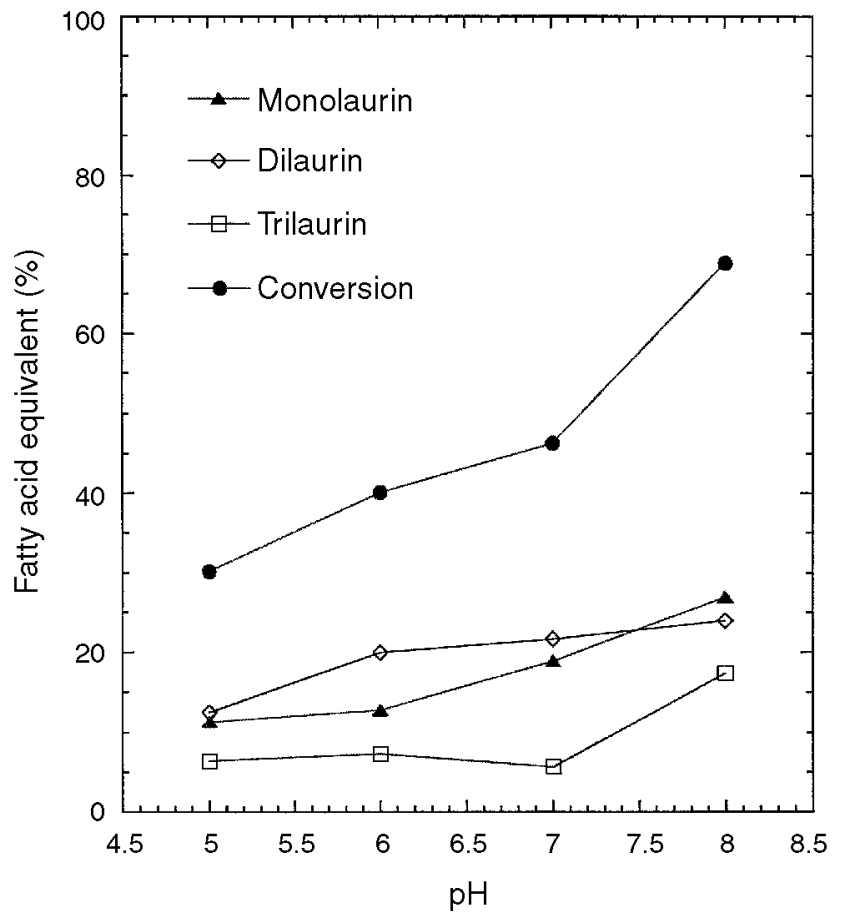

FIG. 6. Effect of $\mathrm{pH}$ on the conversion and product distribution after 24 h. Reaction conditions were $\mathrm{T}=50^{\circ} \mathrm{C}$, flow rate $=20 \mathrm{~mL} / \mathrm{min}$, and buffer concentration $=0.1 \mathrm{M}$.

where stereoselectivity is usually a concern, the formation of TL could be due to the combination of two mechanisms: lack of the strict 1,3-specificity that Lipozyme normally exhibits, and nonenzymatic acyl migration. It has been reported that lipase from $R$. miehei does not have an absolute 1,3-specificity even when reactions take place in a medium where acyl migration is inhibited $(12,21)$. As stated in the literature, acyl migration is accelerated in a buffered or aqueous environment, whereas it is suppressed in low-water-content media $(36,37)$. Characteristics of acyl migration in foam media are currently under investigation.

Effect of ionic strength. We observed that foaming in the buffered solution was relatively stable and that foam height was comparatively high. Reactions conducted in buffer and in deionized water (in which no foaming was observed) achieved conversions of 75 and $21 \%$, respectively. Results from the effect of ions on enzymatic reactions have been reported $(19,38)$. Nonetheless, the mechanism remains unclear, and $\mathrm{pH}$ and ionic strength have not usually been tested separately. We tried to examine the individual effects of these two parameters by fixing the $\mathrm{pH}$ at 7.0. Figure 7 shows the final conversion as well as the rate of esterification of the fatty acid for three different ionic strengths of the buffer solution. Reactions that contain medium phosphate $(0.1-0.15 \mathrm{M})$ concentrations can achieve conversions as high as $76 \%$ after $24 \mathrm{~h}$. On the other hand, at either high (1 M) or low phosphate concentrations $(0.01 \mathrm{M})$, reactions were severely inhibited, leading to yields of 30 and $44 \%$, respectively. In both reactions, little TL was produced. We compared the effect of buffer it- 


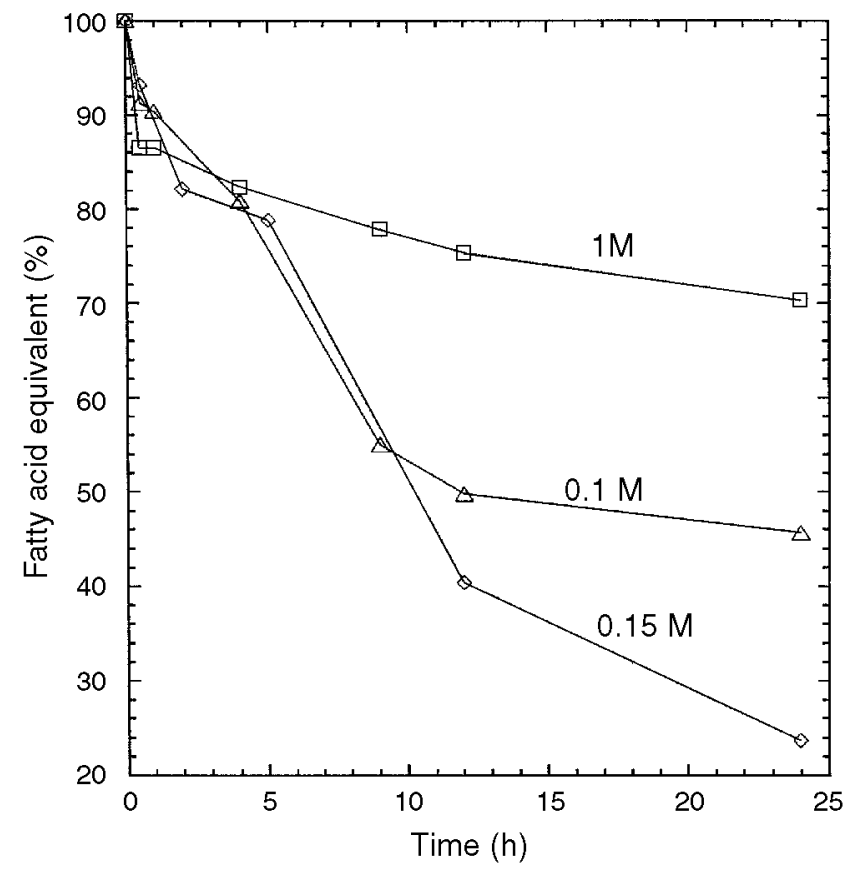

FIG. 7. Effect of buffer concentration on fatty acid conversion. Reaction conditions were: $\mathrm{LA} / \mathrm{GLY}=0.1, \mathrm{~T}=50^{\circ} \mathrm{C}$, flow rate $=20 \mathrm{~mL} / \mathrm{min}, \mathrm{pH}=7$.

self by preparing a $0.1 \mathrm{M}$ phosphate buffer ourselves and testing it against the commercial phosphate buffer of the same strength. We observed some differences between the two, but the difference was not large enough to be conclusive.

When low phosphate concentrations were employed, the mixture did not foam well. We believe that poor foaming is responsible for lower interfacial area and therefore low conversion. If the foams are mostly stabilized by the salt forms (soaps) of fatty acids, the ionic strength moderates the repulsive forces between the negatively charged carboxylic acid head groups. At low ionic strengths, the charge repulsion between the soap molecules increases the surface area per soap molecule and leads to unstable films. Increased ionic strength, on the other hand, leads to closer packing of soap molecules at the air-water interface and to formation of micelles in bulk solution. Both of these processes lead to decreased total interfacial area, and micelle formation reduces the amount of fatty acid available for reaction. The net overall effect is low catalytic efficiency. Figure 7 data show that there is a balance point between head group repulsion and increased micelle formation, for which the rate of reaction is at maximum because of increased total surface area and fatty acid concentration at the interface. Additionally, enzymes undergo denaturation under high ionic strength (19).

Substrate molar ratio. Studies of the substrate molar ratio effect on the product conversion of LA-GLY esterifications gave the results shown in Figure 8. For the range of molar ratios of $[\mathrm{LA}] /[\mathrm{GLY}]$ investigated, we found that $100 \%$ conversion can be achieved at molar ratios below 0.05 . When reactions with substrate molar ratios between 0.1 and 1 were carried out, the conversion was approximately $70 \%$. We did not

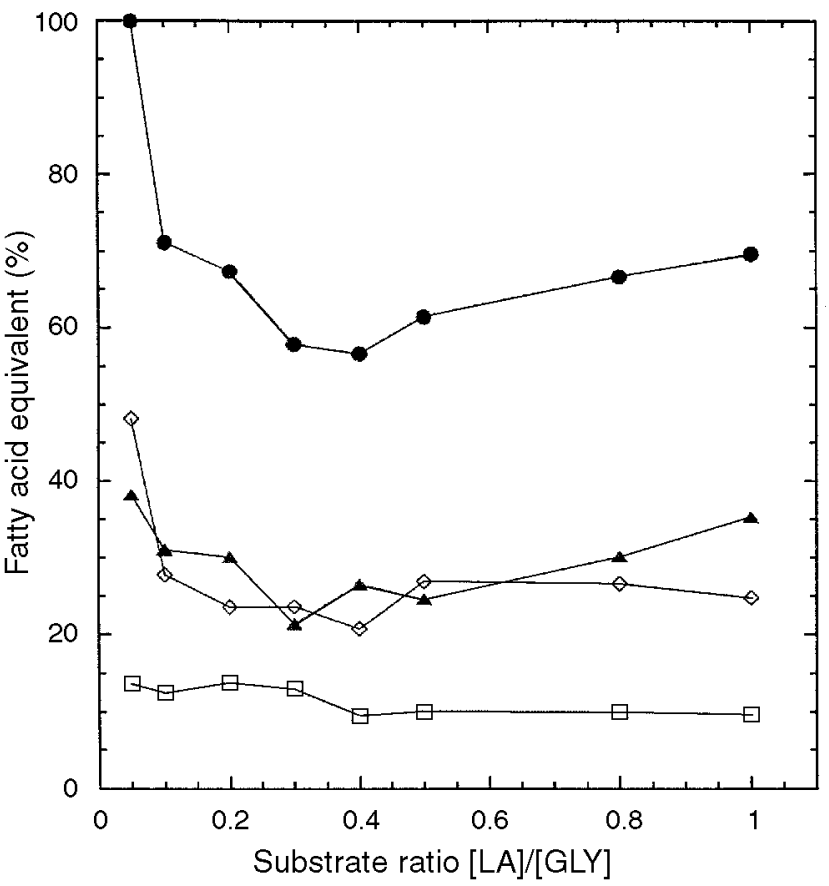

FIG. 8. Effect of substrate molar ratio on the final product distribution after $24 \mathrm{~h}$. Reaction conditions were: $\mathrm{T}=50^{\circ} \mathrm{C}$, air flow rate $=20 \mathrm{~mL} / \mathrm{min}, 0.1$ $\mathrm{M}$ of $\mathrm{pH} 8$ phosphate buffer. Symbols are the same as Figure 2.

observe the substrate inhibition seen in other reaction media $(39,40)$. Even when the substrate molar ratio was as high as 1 , reaction conversion was nearly $70 \%$ after $24 \mathrm{~h}$, with the composition of final product yield being $35.2 \% \mathrm{ML}, 24.8 \%$ DL, and 9.6\% TL. As shown in Figure 9, the normalized rate of consumption of LA was almost independent of the substrate ratio. However, beause we kept the amount of GLY constant, the rate of fatty acid consumption increased linearly with fatty acid concentration.

Based on the results discussed previously, the yield of glyceride synthesis is a strong function of experimental variables. As opposed to what is observed in other media, the main products are always ML and DL in foam media. As discussed in the introduction and shown in Figure 10, there are two important regions that can be identified in foam media: foam films or lamellae, and the Plateau borders where three lamellae meet. Lamellae are assemblies of thin foam films, whereas the Plateau border is where most liquid and excess substance excluded from the thin films reside $(31,32)$. Owing to the Plateau border serving as a reservoir, we believe that, even with a high initial water content in our reactions, the foam films are actually made up of multiple layers of surfaceactive species (including fatty acid, monoglyceride, diglyceride, triglyceride, and enzyme molecules) and a minimal amount of GLY-containing aqueous solution. Presumably, high production of ML and DL is the result of the largely extended interfacial area (between the water and oil phases). Owing to the high surface activity of both fatty acid and 


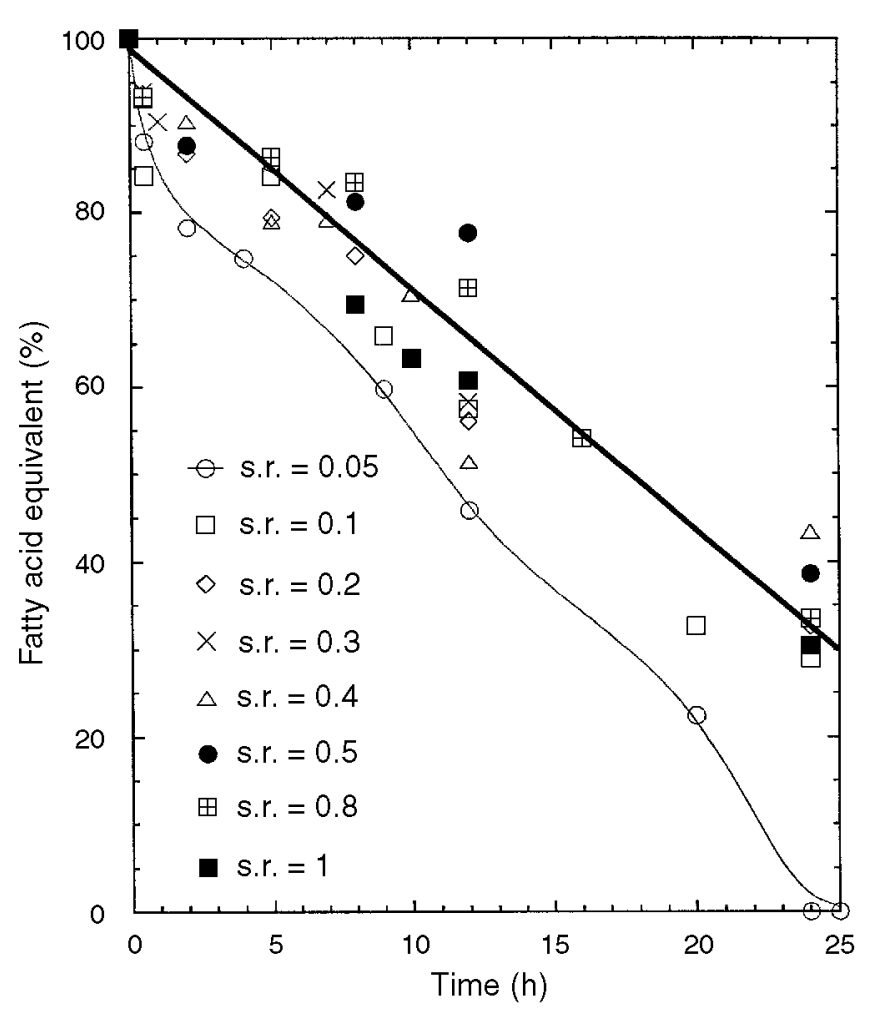

FIG. 9. Effect of substrate molar ratio on lauric acid conversion as a function of time. Reaction conditions were the same as in Figure 8. monoglyceride molecules, they are easily adsorbed onto the films, being subsequently converted into monoglyceride and diglyceride, respectively. Further conversion of DL into TL is comparatively more difficult because of its lower surface activity. Other factors, such as the solubility of products in the reaction mixture and the thermodynamic equilibrium of the product distribution, can also affect the final product composition. Therefore, we believe that the dynamic equilibrium of the foam systems is a result of a delicate balance between partitioning of the reactants in the interfacial layers and in the Plateau borders. Saturation of foam films or deterioration of the drainage can probably lead to reaction inhibition.

We report here glyceride synthesis with an unusually high initial water content (50 wt\%, while other studies are mostly carried out with water content of less than 5\%) (10,38-40). The reactions, nonetheless, favored ester synthesis in which high conversions, especially high production of ML and DL, can be obtained. In conclusion, foam is an excellent medium for converting fatty acid and GLY into mono- and diglycerides. Conversions of $70 \%$ or higher can easily be achieved (within $24 \mathrm{~h}$ ) under optimal operating conditions. The effects of other parameters, such as substrate specificity, the effect of fatty acid chainlength, and other possible reactions, are being investigated.

\section{ACKNOWLEDGMENTS}

Partial support of this research by the National Science Foundation (EID 9018746) is gratefully acknowledged. We thank Novo Inc. for the generous gift of lipase from $R$. miehei (Lipozyme).

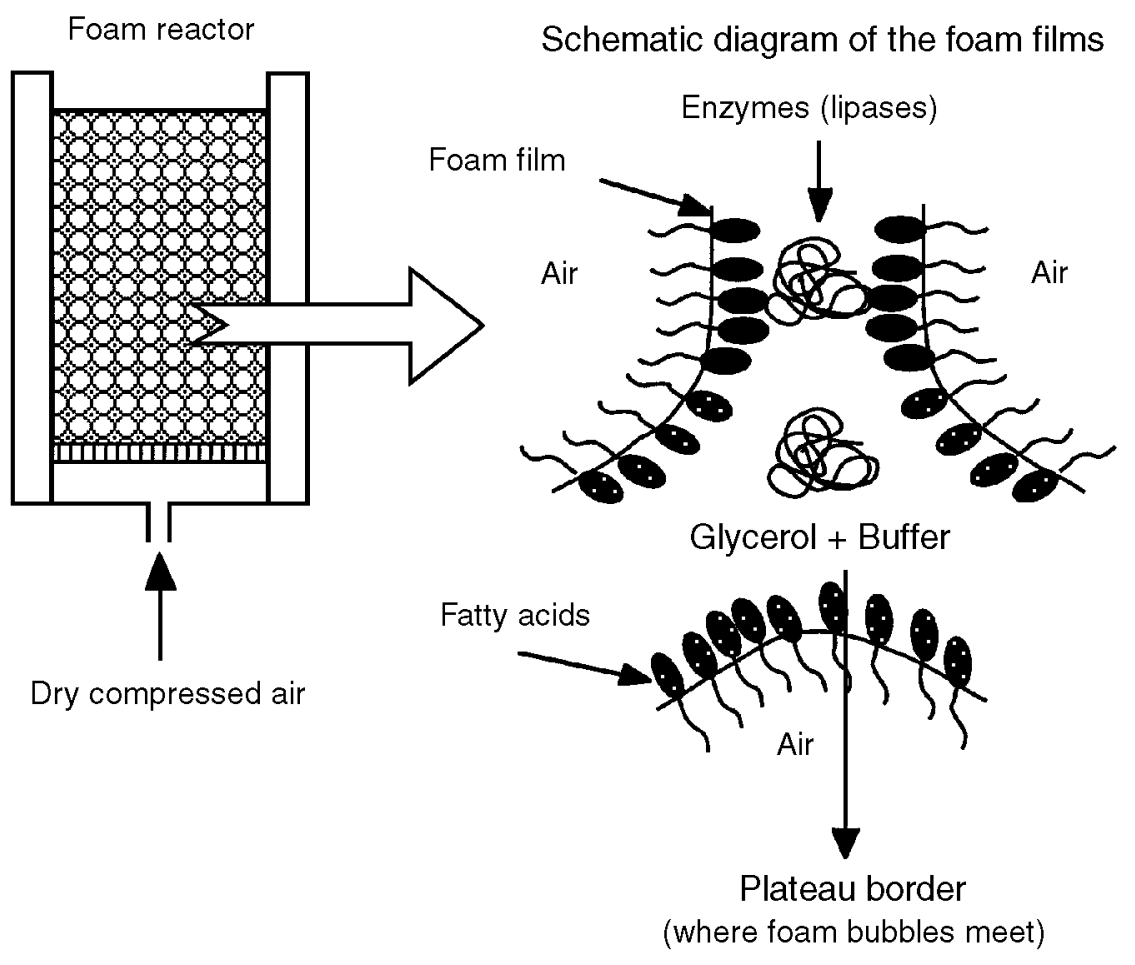

FIG. 10. Schematic diagram of the foam films and locations of various reactants. 


\section{REFERENCES}

1. Margolin, A.L., and A.M. Klibanov, Peptide Synthesis Catalyzed by Lipase in Anhydrous Organic Solvents, J. Am. Chem. Soc. 109:3802-3804 (1987).

2. Therisod, M., and A.M. Klibanov, Facile Enzymatic Preparation of Monoacylated Sugars in Pyridine, Ibid. 108:5638-5640 (1986).

3. Inagaki, M., J. Hiratake, T. Nisioka, and J. Oda, Lipase-Catalyzed Kinetic Resolution with in situ Racemization: One-Pot Synthesis of Optically Active Cyanohydrin Acetates from Aldehydes, Ibid. 113:9360-9361 (1991).

4. Posorke, L.H., G.K. LeFebvre, C.A. Miller, T.T. Hansen, and B.L. Glenvig, Process Consideration of Continuous Fat Modification with an Immobilized Lipase, J. Am. Oil Chem. Soc. 65:6, 922-926 (1988).

5. Extended Summaries International Conference on the Enzymatic Synthesis and Modification of Carbohydrates, J. Chem. Technol. Biotechnol. 56:407-419 (1993).

6. Akoh, C.C., Lipase-Catalyzed Synthesis of Partial Glyceride, Biotechnol. Lett. 15:9, 949-954 (1993).

7. Marangoni, A.G., R.D. McCurdy, and E.D. Brown, Enzymatic Interesterification of Triolein with Tripalmitin in Canola Lecithin-Hexane Reverse Micelles, J. Am. Oil Chem. Soc. 70:737-744 (1993).

8. Pecnik, S., and Z. Knez, Enzymatic Fatty Ester Synthesis, Ibid. 69:261-265 (1992).

9. Seino, H., and T. Uchibori, Enzymatic Synthesis of Carbohydrate Esters of Fatty Acid. (1) Esterification of Sucrose, Fructose, and Sorbitol, Ibid. 71:1761-1765 (1994)

10. Trani, M., F. Ergan, and Q. Andre, Lipase-Catalyzed Production of Wax Esters, Ibid. 68:20-22 (1991).

11. Gillis, A., Research Discovers New Roles for Lipases, Ibid. 65:846-853 (1988).

12. Bhattacharyya, S., and D. K. Bhattacharyya, Biorefining of High Acid Rice Bran Oil, Ibid. 66:1469-1471 (1989).

13. Kosugi, Y., T. Kunieda, and N. Acuma, Continual Conversion of Free Fatty Acid in Rice Bran Oil to Triacylglycerol by Immobilized Lipase, Ibid. 71:445-448 (1994).

14. Berger, M., and M.P. Schneider, Regioselectivity of Lipase in Organic Solvents, Biotechnol. Lett. 13:333-338 (1991).

15. Meusel, D., N. Weber, and K.D. Mukherjee, Stereoselectivity of Lipases: Esterification Reactions of Octadecylglycerol, Chem. Phys. Lipids 61:193-198 (1992).

16. Miller, C.A., H. Austin, L. Posorske, and J. Gonzier, Characteristics of an Immobilized Lipase for the Commercial Synthesis of Esters, J. Am. Oil Chem. Soc. 65:927-931 (1988).

17. Berger, M., K. Laumen, and M.P. Schneider, Enzymatic Esterification of Glycerol I. Lipase-Catalyzed Synthesis of Regioisomerically Pure 1,3-sn-Diacylglycerols, Ibid. 68:955-960 (1992).

18. Berger, M., and M.P. Schneider, Enzymatic Esterification of Glycerol II. Lipase-Catalyzed Synthesis of Regioisomerically Pure 1(3)-rac-Monoacylglycerols, Ibid. 69:961-965 (1992).

19 Hayes, D.G., and E. Gulari, Esterification Reactions of Lipase in Reverse Micelles, Biotechnol. Bioeng. 35:793-801 (1990).

20. Hayes, D.G., and E. Gulari, 1-Monoglyceride Production from Lipase-Catalyzed Esterification of Glycerol and Fatty Acid in Reverse Micelles, Ibid. 38:507-517 (1991).

21. Hayes, D.G., and R. Kleiman, 1,3-Specific Lipolysis of Lesquerella fendleri Oil by Immobilized and Reverse-Micellar Encapsulated Enzymes, J. Am. Oil Chem. Soc. 70:1121-1127 (1993).
22. Forsell, P., P. Parovuori, P. Linko, and K. Poutanen, Enzymatic Transesterification of Rapeseed Oil and Lauric Acid in a Continuous Reactor, Ibid. 70:1105-1109 (1993).

23. Hoq, M.M., T. Yamane, and S. Shimizu, Continuous Synthesis of Glycerides by Lipase in a Microporous Membrane Bioreactor, Ibid. 61:776-781 (1984).

24. Padt, A., M.J. Edema, J.J. Sewalt, and K. Riet, Enzymatic Acylglycerol Synthesis in a Membrane Bioreactor, Ibid. 67:347-352 (1990).

25. Stevenson, D.E., R.A. Stanley, and R.H. Furneaux, Glycerolysis of Tallow with Immobilized Lipase, Biotechnol. Lett. 15:1043-1048 (1993).

26. McNeil, G.P., S. Shimizu, and T. Yamane, Solid Phase Enzymatic Glycerolysis of Beef Tallow Resulting in a High Yield of Monoglyceride, J. Am. Oil Chem. Soc. 67:779-783 (1990).

27. McNeil, G.P., S. Shimizu, and T. Yamane, High Yield Enzymatic Glycerolysis of Fats and Oils, Ibid. 68:1-5 (1991).

28. McNeil, G.P., and T. Yamane, Further Improvements in the Yield of Monoglycerides During Enzymatic Glycerolysis of Fats and Oils, Ibid. 68:6-10 (1991).

29. McNeil, G.P., D. Borowitz, and R.G. Berger, Selective Distribution of Saturated Fatty Acids into the Monoglyceride Fraction During Enzymatic Glycerolysis, Ibid. 69:1098-1103 (1992).

30. Yamane, T., S.T. Kang, K. Kawaharu, and Y. Koizumi, HighYield Diacylglycerol Formation by Solid-Phase Enzymatic Glycerolysis of Hydrogenated Beef Tallow, Ibid. 71:339-342 (1994).

31. Bikerman, J.J. (ed.), Foams, Springer-Verlag, New York, 1973.

32. Oh, S.G., C.P. Singh, and D.O. Shah, Esterification of Stearic Acid with Glycerol by Lipase in Foam, Langmuir 8:2846-2847 (1996).

33. Ergan, F., and G. Andre, Simple High Performance Liquid Chromatography Methods for Monitoring Lipase Reactions, Lipids 24:76-78 (1989).

34. Lutzke, B.S., and J.M. Braughler, An Improved Method for the Identification and Quantitation of Biological Lipids by HPLC Using Laser Light-Scattering Detection, J. Lipid Res. 31:2127-2130 (1990).

35. Liu, J., T. Lee, E. Bobik, M. Guzman-Harty, and C. Hastilow, Quantitative Determination of Monoglycerides and Diglycerides by High-Performance Liquid Chromatography and Evaporative Light-Scattering Detection, J. Am. Oil Chem. Soc. 70:343-347 (1993).

36. Tanaka, T., E. Ono, M. Ishihara, and S. Yamanaka, Enzymatic Acyl Exchange of Triglyceride in $n$-Hexane, Agric. Biol. Chem. 45:2387-2389 (1981)

37. Heisler, A., C. Rabiller, and L. Hublin, Lipase Catalyzed Isomerisation of 1,2-(2,3-) Diglyceride into 1,3-Diglyceride. The Crucial Role of Water, Biotechnol. Lett 13:327-332 (1991).

38. Tahoun, M.K., and H.A. Ali, Specificity and Glyceride Synthesis by Mycelial Lipases of Rhizopus delemar, Enzyme Microb. Technol. 8:429-432 (1986).

39. Yamaguchi, Y., and T. Mase, High-Yield Synthesis of Monoglyceride by Mono-and Diacylglyceride Lipase From Penicillium camemberti U-150, J. Ferment. Bioeng. 72:162-167 (1991).

40. Ergan, F., M. Trani, and G. Andre, Production of Glycerides from Glycerol and Fatty Acid by Immobilized Lipase in Nonaqueous Media, Biotechnol. Bioeng. 35:195-200 (1990).

[Received April 21, 1997; accepted December 8, 1997] 